\title{
Design And Optimization A Circular SHAPE NETWORK ANTENNA MICRO STRIP FOR SOME APPLICATION
}

\author{
Louati Siwar ${ }^{1}$, Fethi Mejri ${ }^{2}$ and Aguili Taoufik ${ }^{3}$ \\ 1,2,3SYS'COM Laboratory, National Engineering School of Tunis, University Tunis El \\ Manar, Tunis, Tunisia
}

\begin{abstract}
To meet the demands of high speed required by mobile communication of past generations, one solution is to increase the number of antennas to the show and the reception of the wireless link this is called MIMO (Multiple input, Multiple output )technology .however, the integration of multiple antennas on the same PCB is delicate because of the small volume that require some applications and electromagnetic antenna between the coupling ,phenomena that we cannot neglect them .indeed a strong isolation between them has been reached to reduce fading of the signal caused by the electromagnetic antenna reached to reduce fading of the signal caused by the electromagnetic coupling and maximize the overall gain in this article we are interested then integration on the same printed circuit of eight antennas MIMO are not operation in the same frequency band the first antenna of this last work at $2.4 \mathrm{GHz}$.other antennas have resonance frequency folling each with $20 \mathrm{MHz}$ offset this device is characterized by its original form that keeps is highly isolated antennas from the point of view electromagnetic coupling
\end{abstract}

\section{INDEX TERMS}

MIMO, Technology Micro-strip, Microwave, Network Antenna

\section{INTRODUCTION}

The research in the field of antennas dies goes back decades ,but is that recently that the use of multiple antennas at both the transmitter and the receiver has shown a marked improvement in the ability of the communications the MIMO channels, which can represent various environments of information transmission, the help of Vector algebra where The popularity of this modelbut quickly a debit or scope problem is presented a new technique appeared in 1984 thanks to Mr..jack winters, who filed a patent for wireless communication to multiple antennas [10]Since this discovery ,this system has been very used in servel areas these of multiple antennas to the emission and reception in radio systems is one of the most important advances developed last times they allow to reduce the likelihood of error and to increase the transmission rate without increasing power transmission or used bandwidth.however , the majority of studies are too theoretical, and currently are there are few rigs to check the viability of these techniques in real transmission conditions and get a more realistic assessment of the performance of the system In recent developments in telecommunications ,the multiple technology uses spatial diversity .it was already this principle on the Wi-Fi systems legacy the addition of receiver antennas allowed for example to make a selection of the antenna on a criterion of power to take micro strip antennas 
deep applications especially in the filed mobile military, medical and telecommunication. 'We theCapacity of Radio Communication Systems with Diversity in a Rayleigh Fading Environment "[11], and "The Diversity Gain of Transmit Diversity in Wireless Systems with Rayleigh Fading "[11]. We will proceed with a review of the principles: basic necessary to the understanding of the articles,followed by analysis and reproduction of the results of the two sections of winters MIMO Technology using networks of antennas on the show or at the front desk in order to improve the quality of the signal to noise. the allows also to decrease the level of radio signals in the life of batteries in the case of a telephone MIMO antennas ,space diversity spatial multiplexing: stream emitted at the same time , on the same frequency can be considered three main categories of MIMO: space diversity MIMO: simultaneously Sent a message on different antennas to the show signals received on each of the receiving antennas are then released phase and ordered in a consistent Manner .a simplified version uses the signal of only one of the antennas, one that gets the best signal at a time given (polarized antennas) it allows to increase the ratio signal /noise (thanks to the diversity gain) of the transmission .for this technique to be effective ,must the MIMO sub channels are uncorrelated (independent)from the other MIMO spatial multiplexing :each message in cut in sub messages. . At the same time passed the different sub messages on each of the transmit antennas. The signals received on the receiving antennas are reassembled to reconstruct the original message as for diversity MIMO; the sub channels of propagation must be uncorrelated MIMO multiplexing to increase rates of transmission (through multiplexing gain) .diversity and multiplexing MIMO techniques can be applied jointly

\section{DESIGN METHODOLOGY}

A circular micro strip patch antenna designing is easier than other other patch configuration as we only need one design parameter i; e radius of the patch in the procedure of typical Micro strip "the frequency of resonance desired antenna design The micro strip structure that we used has a driver in copper characterized by a conductivity $\zeta=58,5.106(\mathrm{~S} / \mathrm{m})$ and a thickness $\mathrm{T}=35 \mu \mathrm{m}$. the substrate is glass Teflon of relative permittivity $\varepsilon r=4.32$ thickness $\mathrm{H}=1,5 \mathrm{~mm}$ and has losses modeled by tag $=0$, 0019. was used ADS as a software Simulation of an antenna We used as software ADS as a simulation softwarethen a patch antenna which works at the frequency of operation specified $\mathrm{f} 0=2 \mathrm{GHz}$ et $3 \mathrm{GHz}$ can be designed using the equations of the model line transmission [5] insertion of supply type used as a feedingmethod formulas used for calculating radius $\mathrm{R}$ or (a)

$$
\begin{gathered}
a=F\left\{1+\frac{2 h}{\pi F \varepsilon_{r}}\left[\ln \left(\frac{\pi F}{2 h}\right)+1.7726\right]\right\}^{-1 / 2} \\
F=\frac{8.791 \times 10^{9}}{f_{r} \sqrt{\varepsilon_{r}}}
\end{gathered}
$$




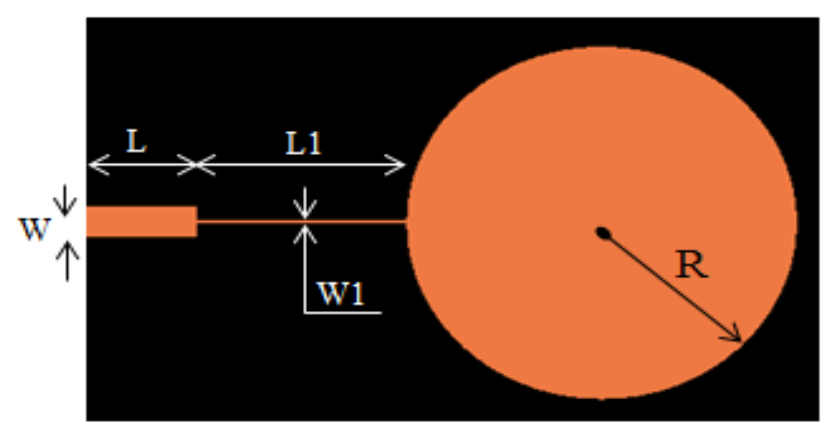

Fig1:Schematic of a Circular micro strip patch antenna

The procedure assumes that the specified information includes the dielectric constant of the substrate $(\varepsilon r)$, the operating frequency $(f)$ and the height of the substrate $(h)$. To find the actual radius ' $a$ ' In the above equations we should remember that the operating frequency $f$ should be taken in Hertz $(\mathrm{Hz})$ and the height of the substrate $h$ should be taken in Centimeters $(\mathrm{cm})$

\subsection{DeSIGN An ANTENNA}

The antenna represent an important component of the chain of transmission since it affects the transmission and reception of the signal radiated in free space the choice of type of antenna depends on type of network of covered area and emission levels to do this you choose a circular antenna We used as software ADS as a simulation software.An antenna is connected to the source by a characteristic impedance transmission line $Z_{-} c\left(Z_{-} c=50 \mathrm{Ohm}\right)$.to ensure maximum transfer of power between power and antenna ,it is necessary to ensure an impedance matching the adaptation allows canceling the coefficient of reflection $S(11)$ the antenna input .The reflection coefficient is the ratio of the reflected antenna input wave and the incident wave.it depends on the antenna and the characteristic impedance. The characteristic impedance of the line quart wave which must be intercalated between the patch and 50ohm line is calculated with the following formula:

$$
Z_{\lambda \mathrm{g} / 4}=\left(Z_{50 \text { ohms }} \cdot Z_{e}\right)^{1 / 2}
$$

After a simulation, we notice that the antenna is well suited as mitigation of $\mathrm{dB}$ which is strictly lower value $\mathrm{DB}$ and which represents a minimum reflection of the reports mean notes will be transmitted, there is also an impedance value is equivalent to $62 \mathrm{Ohm}$ for $2.4 \mathrm{GHz}$ frequency. These results are show in figure values :L1 $=17.02 \mathrm{~mm}$ of circular antenna $\mathrm{L} 2=18.62 \mathrm{~mm}, \mathrm{~W} 2$ $=0.198 \mathrm{~mm}, \mathrm{~L} 3=10 \mathrm{~mm}$ and $\mathrm{W} 3=2.86 \mathrm{~mm}$ 
International Journal of Computer Science \& Information Technology (IJCSIT) Vol 9, No 1, February 2017

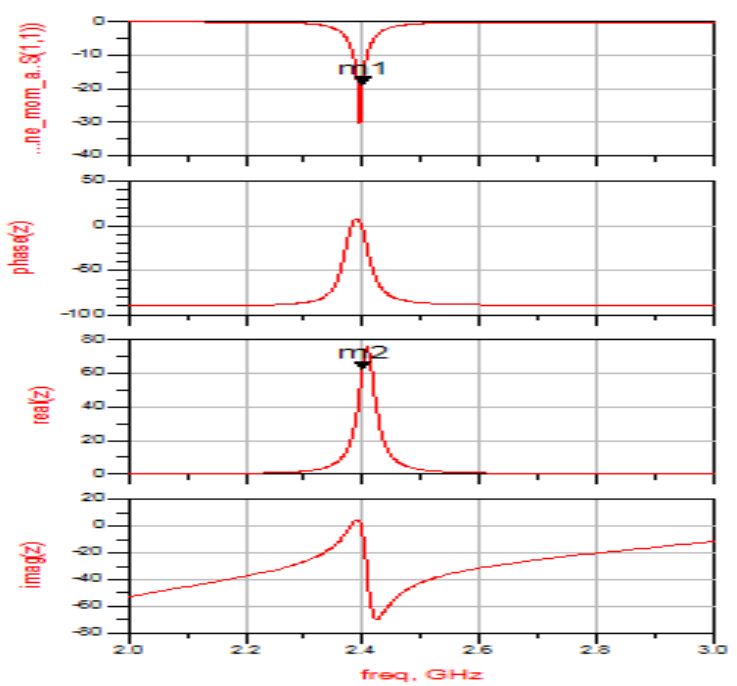

Fig 2: results of simulation of the antenna under Momentum (S11 (dB), phase (Z), real (Z), imag (Z))

Under schematic, we bring the antenna creates in order to, optimize length of line transmission used on the frequency interval[2GHz,3GHz]to do this, we define the lower interval of optimization for the length as well as the coefficient of reflection $\mathrm{S} 11$ under schematic at $-20 \mathrm{~dB}$ after optimization, we get the following for $2.4 \mathrm{GHz}$ frequency. These results are show in figure

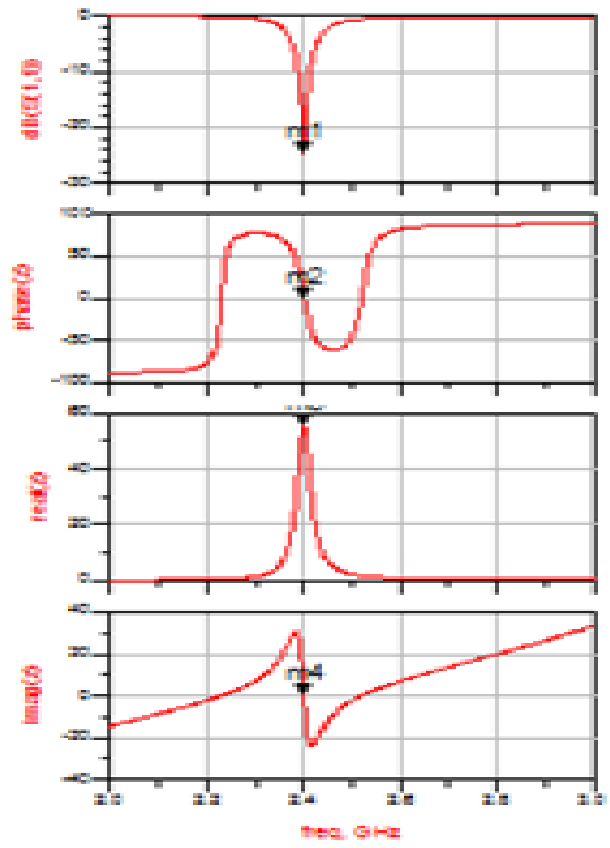

Fig3: results of simulation of the antenna under Momentum (S11(dB), phase $(Z)$, real $(Z)$, imag $(Z))$

All After a simulation, we notice that the antenna is well suited since we have an attenuation of $24.518 \mathrm{~dB}$ Which is la lower value strictly at-20dB and represents a minimum reflection of the signal .we find also an impedance value equal $56.223 \mathrm{Ohm}$ for $2.4 \mathrm{GHz}$ frequency .these results 
are shown in figure 4 Which shows that almost a perfect feed configuration is researched, i.e. The figure represents the first antenna pattern, it indicates that this circular antenna is directive

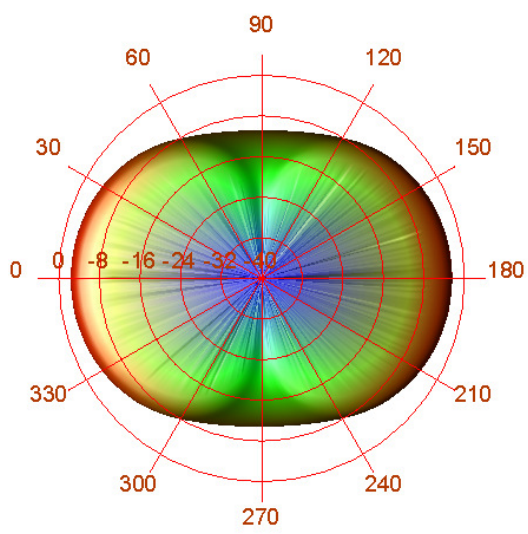

Fig4: 2D (rear view) radiation pattern

the figure represents the first antenna radiation pattern, it indicates that this circular antenna is directivity .it connects our first antenna network analyzer on using a single port, onuses the matter it retrieve the resultant of S11to compare it with the result at that was found by ADS. In this part and after the completion of measures drawing the representative gain curve $S$ (11) and then compared with that of the ADS. This is the result that was found the comparison between the values of $(\mathrm{dB})$ measured and simulated a circular patch on the $2.4 \mathrm{GHz}$ frequency antenna:The figure below shows results found for the $S 11(\mathrm{~dB})$ parameter by simulation under ADS to the resonant frequency are consistent with those found by measures.

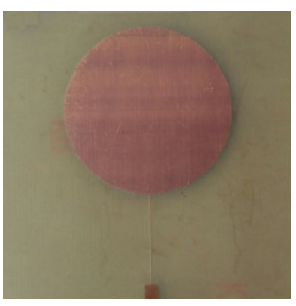

Fig5:After circular patch antenna

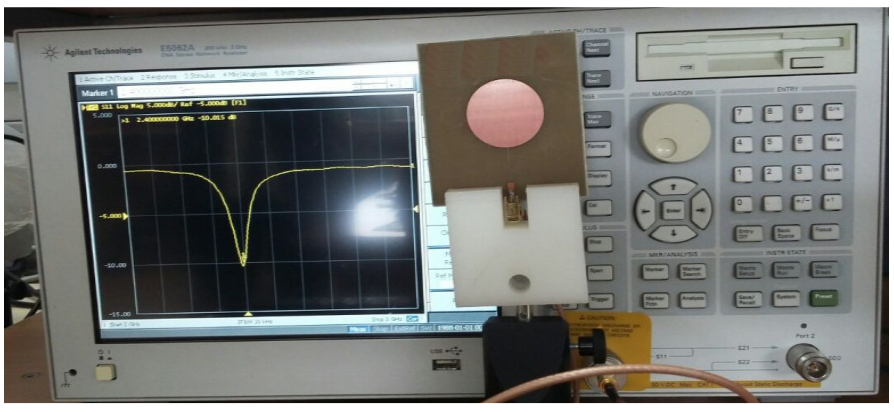

Fig6: measures $\mathrm{S}(11)$ antenna 
International Journal of Computer Science \& Information Technology (IJCSIT) Vol 9, No 1, February 2017

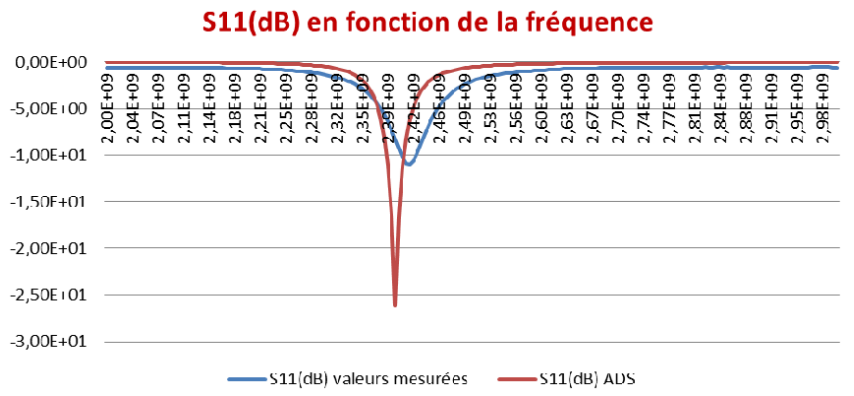

Fig8: Comparison between measured and simulated values S11 (dB)

According to this curve there is that the measures made by the network for this antenna Analyzer are accurate so our work already done by theADS (Advanced DesignSystem) is consistent with th e measures carried out

\section{DESIGN A NETWORK}

Now after the first antenna design was completed other antennas similarly except that by changing the frequency of work the end of all antennas, we turn to the creation network antenna working in $2.4 \mathrm{GHz}$ maximum bandwidth of $20 \mathrm{MHzNow}$ for the solution you made this design is based on the location of circular wave of these 8 antennas ,We'll keep increasing order of these last ,mean we start with the first antenna of frequency $2.4 \mathrm{GHz}$ until you research the last one which is set to the $2.56 \mathrm{GHz}$ frequency .in keeping with , the same frequency offset which is $\Delta \mathrm{f}=$ $20 \mathrm{MHz}$ the first place we fixed the distance from each antenna to the center of the circle that represents the shape of this design such as $\mathrm{R}=50 \mathrm{~mm}$ to set the radius of this circle used GAP that a component of the library of ADS allows to separate the different antennas In our case we have 8 antennas which are placed in an orderly way mean the 8 are separated by an angle $=45$ degres.For this the transport capacity of the used frequency band is increased 16 times $\mathrm{c}$ omparedto theold form that is the parallel formatting.

We take the first three antennas such as theangle between the first and the third equal 90 degrees $\mathrm{s}$ o the first antenna has two forms of polarization (vertical or horizontal in the same she can use the second antenna for that ability the first antenna during transport is increased four times the initial form for the second antenna and the third.)The same treatment for other antennas $(3,4,5)$ by foll ows her $(5,6,7)$ and $(7,8,1)$ Involving 4 groups ofantennas multiplied 4 times so the ability incre ases 16 times.It's the most important thing we did to justify our choice and the characters made by this configuration we'll study it later. 
International Journal of Computer Science \& Information Technology (IJCSIT) Vol 9, No 1, February 2017

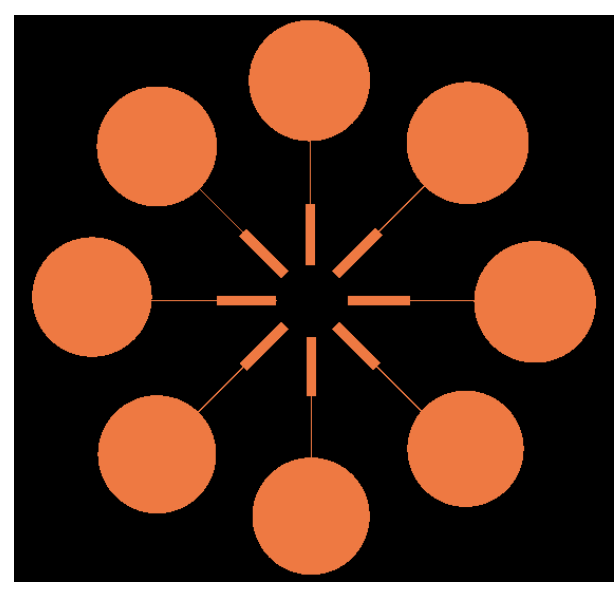

Fig 9: A network antennas under Momentum

After this configuration we realize this network simulation to determine the parameters and the ga in and directivity using ADS

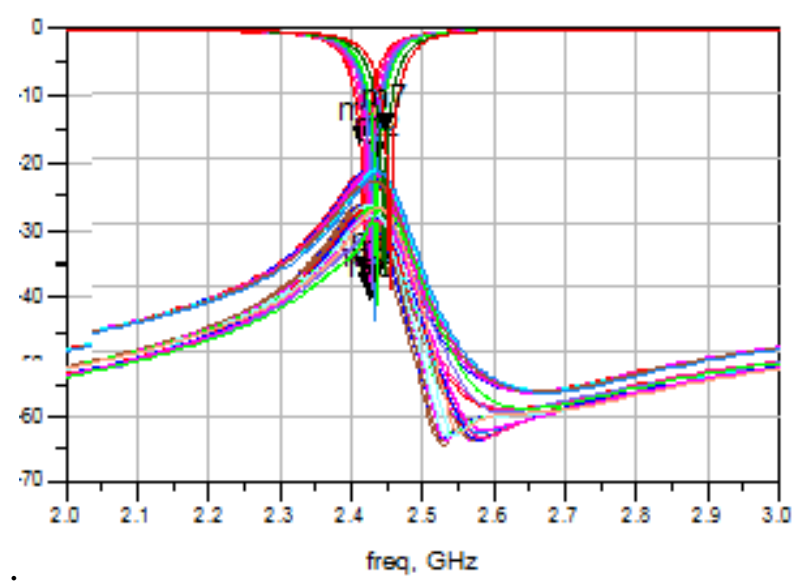

Fig10: Representative curve of theConfiguration

From this placed above figure we notice that the transmission is maximum and that the reflection is minimal, so features are better than the second solution except that for this solution the coupling increases completely but the curves are well separated.

So each antenna will really like this isolated in space where the result sought to establish, even cu rves are better suited for example to the antenna $5=\mathrm{S} 55-41.88$.

After thestudy of sparameters and the gain we turn now to the radiation pattern and directivity me an direction of current during activation of this network after the simulation of network finds the phase shift between different resonant frequencies 


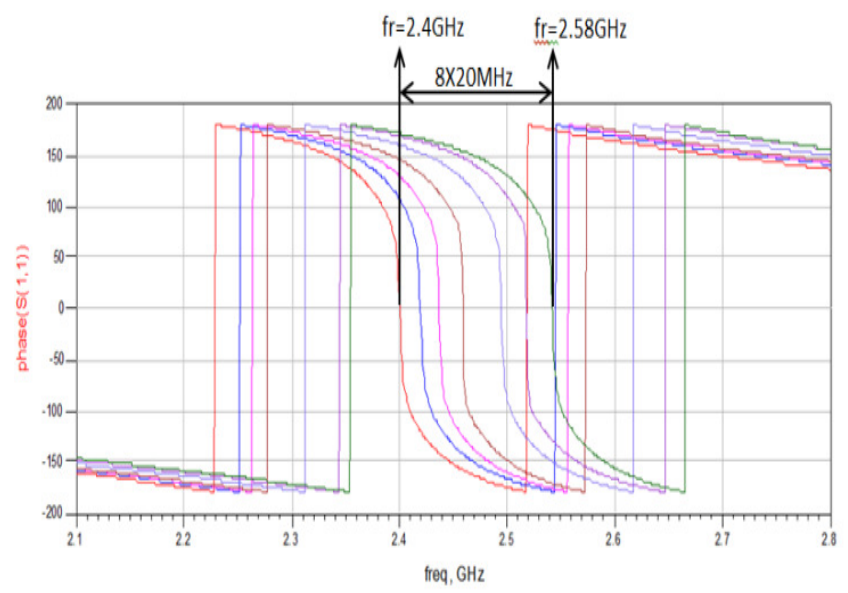

Fig11: results of simulation of the network antennas under Momentum (phase (Z))

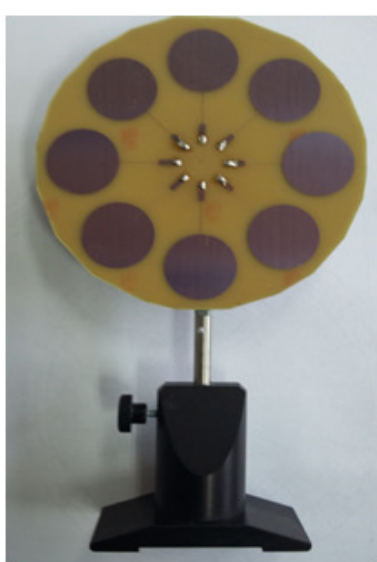

(A)

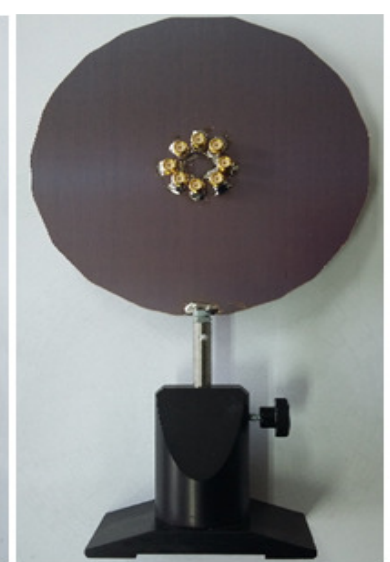

(B)

Fig12: Aerial network realized (A) front view - view from behind (B)

From these representative curves of measures found by the parser we note that ADS gives good results, for this reason we valid this work and we fin is hour designs only with this tool Deferens steps to achieve a suitable antenna design was studied i $\mathrm{n}$ this article and function in a just manner has a specific frequency, and these steps are the as me that We follow when we will determine other antennas except that it changes the frequency of work from one to the other, then completed the measures for the first antenna that was already prepared by the ADS and the validation of the latter were assured. Subsequently measured MIMO network by the Network Analyze $r$ and compared the results found with that it has already carried out by our software, and end of this comparison it concludes that this design is valid

\section{Conclusion}

In this paper, we have presented the design and simulation of a Circular Micro strip patch Antenna at $\mathrm{c}$ band frequency region. The simulated results of circular micro strip patch antenna shows good directivity and gain value. The directivity and gain for the circular patch antenna is 
International Journal of Computer Science \& Information Technology (IJCSIT) Vol 9, No 1, February 2017

$4.5 \mathrm{dBi}$ and $2.66 \mathrm{dBi}$ respectively and a percentage bandwidth achieved is $3.79 \%$. The high return loss value gives perfect impedance matching between patch and feed. Also, designed circular patch antenna gives radiation efficiency equal to $59.1 \%$. However, we can also improve the antenna characteristics using different known technologies to increase the applicability of this patch antenna. Although, this circular patch antenna may find applications satellite communication applications such as transponders

\section{REFERENCES}

[1] Design", Third Edition, John Wiley \& Sons, Inc. [2] Ramesh garg, PrakashBhartia, InderBahl andApisakIttipiboon,Microtrip Design Antenna Handbook", Artech House, Boston London.

[2] Kin-Lu Wong, "Compact and Broadband Microstrip Antennas", John Wiley \& Sons, Inc.

[3] A.Al-Zoubi, F. Yang, and A. Kishk, "A broadband center-fed circular patch-ring antenna with a monopole like radiation pattern," IEEE Transaction Antennas Propagation, vol. 57, pp. 789-792, 2009.

[4] Prasanna L. Zade,SachinS.Khade, Dr. N. K. Choudhary, "Modeling and Designing of Circular Microstrip Antenna For wireless communication", International Conference on Emerging Trends in Engineering and Technology, ICETET- 2009.

[5] Arun Singh Kirar, Veerendra Singh Jadaun, Pavan KumarSharma, "Design a Circular Microstrip Patch Antenna for Dual Band", International Journal of Electronics

Communication and Computer Technology (IJECCT) Volume 3 Issue 2 March 2013.

[6] V.Harsha Ram Keerthi, Dr.Habibullah Khan, Dr.P.Srinivasulu,

"Design of C-Band Microstrip Patch Antenna for Radar Applications Using IE3D", Journal of Electronics and Communication Engineering, 2013.

[7] M.Venkata,Narayana, Govardhani.Immadi, K.Rajkamal, M.S.R.S Tejaswi, "Microstrip Patch Antenna for C-band RADAR

[8] Applications with Coaxial fed", International Journal of Engineering Research and Applications, Vol. 2 , I

[9] Jack H. Winters, 'On the Capacity of Radio Communication Systems with Diversity in a, Rayleigh Fading Environment", IEEE JOURNAL ON SELECTED AREAS IN COMMUNICATIONS, VOL. SAC-5, NO. 5, JUNE 1987, p871

[10] Jack H. Winters, 'The Diversity Gain of Transmit Diversity in Wireless Systems with Rayleigh Fading", IEEE TRANSACTIONS ON VEHICULAR TECHNOLOGY, VOL. 47, NO. 1, FEBRUARY 1998, p119 